\title{
Análise de convergência espacial dos repasses da Lei Robin Hood ${ }^{1}$
}

\author{
Noé Gonçalves Maranduba Júnior ${ }^{2}$ \\ Eduardo Simões de Almeida ${ }^{3}$
}

\section{Resumo}

Alega-se que a Lei Robin Hood tem permitido uma melhor distribuição dos valores do ICMS devidos aos municípios do estado de Minas Gerais. O objetivo deste artigo é investigar se essa lei, entre os anos de 2001 e 2005, realmente foi eficaz, isto é, se, em termos relativos, municípios pobres receberam mais dessas transferências do que os municípios ricos. Para fazer isso metodologicamente, implementa-se uma análise exploratória de dados espaciais e uma análise de convergência, para verificar se as disparidades nos repasses diminuíram com o passar do tempo. Os resultados mostraram que os efeitos espaciais importaram nas análises e que não houve um efeito redistributivo dos repasses, considerando-se que o coeficiente indicador de convergência não foi significativo.

Palavras-chave: Lei Robin Hood; Dados espaciais - Análise exploratória; $\beta$ convergência; Econometria espacial.

\section{Abstract \\ Spatial convergence analysis of tax transfers from the Robin Hood Act}

One alleges that the Robin Hood Act has allowed one better distribution of the values of ICMS to municipalities of the state of Minas Gerais. The paper is aimed at verifying if the Robin Hood Act has actually revealed a redistributive effect in tax transfers to municipalities in the state over the period 2001-2005, that is, if, in relative terms, poor municipalities have received more these transfers than rich ones. In doing methodologically so, an exploratory spatial data analysis and a convergence analysis are implemented to verify if the disparities of tax redistribution have diminished over the time. The findings showed that the spatial effects were important in the analysis as well as there was no redistributive effect in the period because the convergence coefficient was not significant.

Key words: Robin Hood Act; Exploratory spatial data analysis; $\beta$ convergence; Spatial econometrics.

JEL C21.

\section{Introdução}

O estado de Minas Gerais, devido à sua dimensão geográfica e grande heterogeneidade socioeconômica de suas regiões, requer permanentes estudos para

(1) Trabalho recebido em março de 2007 e aprovado em outubro de 2008.

(2) Mestrando em Economia Aplicada da Faculdade de Economia e Administração da Universidade Federal de Juiz de Fora (FEA//UFJF), Juiz de Fora, MG, Brasil. E-mail: noegmj@ gmail.com.

(3) Bolsista de Produtividade em Pesquisa do CNPq - Nível 2 / Professor adjunto da Universidade Federal de Juiz de Fora (UFJF), MG, Brasil. O autor agradece o apoio e o financiamento para a elaboração deste artigo do CNPq, Fapemig e UFJF. E-mail: edualmei@gmail.com. 
se avaliar a eficácia de políticas regionais adotadas, como é o caso daquelas de distribuição de recursos tributários visando à equidade social.

Na Constituição Federal de 1988 foram instituídas alterações no campo tributário, visando elevar a participação dos municípios na distribuição dos recursos oriundos da receita tributária da União e dos estados. Em seu artigo 158, inciso IV, dispôs-se que 25\% da arrecadação do ICMS (Imposto sobre Operações Relativas à Circulação de Mercadorias e sobre Prestação de Serviços de Transporte Interestadual e Intermunicipal e de Comunicação) pertenceria aos municípios. Em seu artigo 159, § $3^{\circ}$, estabeleceu-se, ainda, que os municípios também teriam direito a $25 \%$ dos recursos recebidos pelos estados, nos termos do artigo 159 , inciso II. $^{4}$

Almejando estabelecer critérios de repartição dos citados recursos entre os municípios, a Constituição Federal determinou em seu artigo 158, parágrafo único, e no seu artigo 159, $\S 3^{\circ}$, que isso fosse efetuado observando-se a seguinte proporcionalidade: $75 \%$, no mínimo, na proporção do valor adicionado (VAF) nas operações relativas ao ICMS, realizadas nos territórios dos municípios e até $25 \%$, conforme dispusesse lei estadual.

Inicialmente, em Minas Gerais ficou estabelecido que a distribuição da cota-parte do ICMS dos municípios observaria três critérios: o Valor Adicionado Fiscal, os Municípios Mineradores e Compensação Financeira por Desmembramento de Distrito, o que se deu através do Decreto-Lei n. 32.771, de julho de 1991. A distribuição realizada com base nesses critérios, no entanto, demonstrava um alto grau de concentração de recursos nos municípios mais desenvolvidos e ativos economicamente, possuidores de maior volume de VAF. Diante disso, a Lei Estadual n. 12.040 de 28/12/95 (conhecida como Lei Robin Hood, haja vista o personagem inglês do século XII que roubava a riqueza dos nobres e a distribuía aos necessitados) indicava novos critérios visando descentralizar a distribuição da cota-parte do ICMS dos municípios, para beneficiar os municípios que investissem em educação, saúde, produção de alimentos, conservação do patrimônio histórico e cultural e preservação do meio ambiente, bem como realizassem esforços na arrecadação tributária própria.

Em dezembro de 1996 foi publicada a Lei n. 12.428 que alterou a lei anterior, melhorando a participação de critérios como: área geográfica, população, população dos cinquenta (50) mais populosos, educação, saúde, meio ambiente, patrimônio cultural, produção de alimentos e receita própria.

(4) Isso determinou que os estados recebessem da União $10 \%$ da arrecadação do Imposto sobre Produtos Industrializados (IPI), proporcional ao valor das respectivas exportações de produtos industrializados. 
Em 27 de dezembro de 2000, foi aprovada a Lei n. 3.803, a qual revogou e substituiu a anterior (12.428/96). A nova lei estabeleceu critérios e porcentuais que abrangem o período analisado neste artigo (do ano de 2001 até 2005).

Conforme se pode observar no Quadro 1, a seguir, dentre os critérios da Lei Robin Hood se encontra o do VAF (art. $1^{\circ}$, I). Trata-se, na verdade, de opção realizada pelo estado de Minas Gerais no sentido de integrá-lo como um dos critérios de distribuição da Lei Robin Hood, mas não de exigência constitucional.

O cálculo dos valores a serem repassados aos municípios é complexo, com a utilização de índices cuja periodicidade é mensal, trimestral e anual, envolvendo os critérios listados no Quadro 1: valor adicionado fiscal (VAF), receita própria dos municípios, gastos com saúde, meio ambiente, patrimônio cultural, produção de alimentos, educação, área geográfica, população e população dos 50 mais populosos, cota mínima e municípios mineradores. As análises a serem efetuadas neste trabalho dizem respeito à totalidade dos repasses (os 25\%).

Quadro 1

Critérios de distribuição da Lei Robin Hood, no período de 2000 a 2005

\begin{tabular}{|c|c|c|c|c|c|c|}
\hline Critérios de Distribuição & 2000 & 2001 & 2002 & 2003 & 2004 & 2005 \\
\hline VAF $\left(\right.$ art. $\left.1^{\circ}, \mathrm{I}\right)$ & 4,615 & 4,632 & 4,644 & 4,656 & 4,668 & 4,680 \\
\hline Área geográfica $\left(\right.$ art. $\left.1^{\circ}, \mathrm{II}\right)$ & 1,000 & 1,000 & 1,000 & 1,000 & 1,000 & 1,000 \\
\hline População (art. $1^{\circ}$, III) & 2,710 & 2,710 & 2,710 & 2,710 & 2,710 & 2,710 \\
\hline $\begin{array}{l}\text { População dos } 50 \text { mais populosos (art. } \\
\left.1^{\circ}, \mathrm{IV}\right)\end{array}$ & 2,000 & 2,000 & 2,000 & 2,000 & 2,000 & 2,000 \\
\hline Educação (art. $\left.1^{\circ}, \mathrm{V}\right)$ & 2,000 & 2,000 & 2,000 & 2,000 & 2,000 & 2,000 \\
\hline Produção de alimentos (art. $1^{\circ}$, VI) & 1,000 & 1,000 & 1,000 & 1,000 & 1,000 & 1,000 \\
\hline Patrimônio cultural (art. $1^{\circ}$, VII) & 1,000 & 1,000 & 1,000 & 1,000 & 1,000 & 1,000 \\
\hline Meio ambiente (art. $\left.1^{\circ}, \mathrm{VIII}\right)$ & 1,000 & 1,000 & 1,000 & 1,000 & 1,000 & 1,000 \\
\hline Gastos com saúde (art. $\left.1^{\circ}, \mathrm{IX}\right)$ & 2,000 & 2,000 & 2,000 & 2,000 & 2,000 & 2,00 \\
\hline Receita própria (art. $\left.1^{\circ}, \mathrm{X}\right)$ & 2,000 & 2,000 & 2,000 & 2,000 & 2,000 & 2,00 \\
\hline Cota mínima (art. $\left.1^{\circ}, \mathrm{XI}\right)$ & 5,500 & 5,500 & 5,500 & 5,500 & 5,500 & 5,5000 \\
\hline Municípios mineradores $\left(\operatorname{art.} 1^{\circ}, \mathrm{XII}\right)$ & 0,110 & 0,110 & 0,110 & 0,110 & 0,110 & 0,110 \\
\hline Mateus Leme (art. $\left.1^{\circ}, \mathrm{XIII}\right)$ & 0,045 & 0,032 & 0,024 & 0,016 & 0,008 & 0,000 \\
\hline Mesquita (art. $\left.1^{\circ}, \mathrm{XIII}\right)$ & 0,019 & 0,016 & 0,012 & 0,008 & 0,004 & 0,000 \\
\hline Total & 25,000 & 25,000 & 25,000 & 25,000 & 25,000 & 25,000 \\
\hline
\end{tabular}

Vale informar que o critério "municípios mineradores" objetiva compensar os municípios mineradores através de uma porcentagem média do extinto IUM (Imposto Único sobre Minerais), recebido pelos municípios no 
Noé Gonçalves Maranduba Júnior / Eduardo Simões de Almeida

exercício de 1988. Ainda, os recebimentos dos municípios de Mateus Leme e Mesquita dizem respeito à compensação financeira por desmembramento de distritos que se emanciparam.

A competência de apuração e distribuição dos valores envolve vários órgãos, dentre os quais a Secretaria de Estado da Fazenda de Minas Gerais, a Fundação João Pinheiro, o Tribunal de Contas do Estado de Minas Gerais e diversas outras secretarias e órgãos estaduais.

Na Figura 1, a seguir, tem-se um esboço da distribuição dos recursos da Lei Robin Hood. O que se observa é que os valores parecem estar distribuídos aleatoriamente no espaço, não se podendo realizar outras afirmativas, haja vista que as três faixas (alta, média e baixa) dos recursos parecem estar presentes em todos os quadrantes do estado.

Há, assim, a necessidade da utilização de técnicas mais apropriadas que a simples inspeção visual de mapas. Assim, como objetivo, aqui se propõe conhecer, para o período de 2001 a 2005, a distribuição espacial dos repasses da Lei Robin Hood bem como testar a hipótese da existência de convergência dos mesmos, checando se tal lei teve efetivamente efeitos redistributivos, ou seja, descobrir se, em termos relativos, as transferências para os municípios pobres cresceram mais do que aquelas para os municípios ricos. Averiguar a convergência dos repasses da Lei Robin Hood é relevante para saber se tal lei está sendo eficaz na sua missão de ter um efeito redistributivo dos recursos fiscais no Estado de Minas Gerais. Para esse fim, utilizam-se a análise exploratória de dados espaciais (Aede) e a análise de convergência espacial. A Aede permite uma análise mais estática, enfocando as distribuições espaciais em dois pontos no tempo, 2001 e 2005. Poder-se-ia dizer que a Aede tira duas fotografias dos repasses da Lei Robin Hood. Por isso, é importante ser complementada pela análise de convergência que indica como está sendo a evolução dinâmica desses repasses ao longo do período completo (2001 até 2005).

Recentemente, conforme pode ser lido em Minas Gerais (2007), os municípios mineiros disputam esses recursos, estando em tramitação na Assembleia Legislativa de Minas Gerais o Projeto de Lei n. 637/07, chamado de ICMS Solidário, que visa estabelecer alteração de critério, de forma a que mais recursos do bolo a ser distribuído sejam transferidos para os municípios mais pobres. Assim sendo, os resultados deste artigo podem servir de subsídios iniciais para o debate que está correntemente sendo travado na Assembleia sobre a necessidade ou não da modificação do critério de distribuição desses recursos. 
Figura 1

Mapa dos repasses da Lei Robin Hood para os municípios mineiros (Ano 2000)

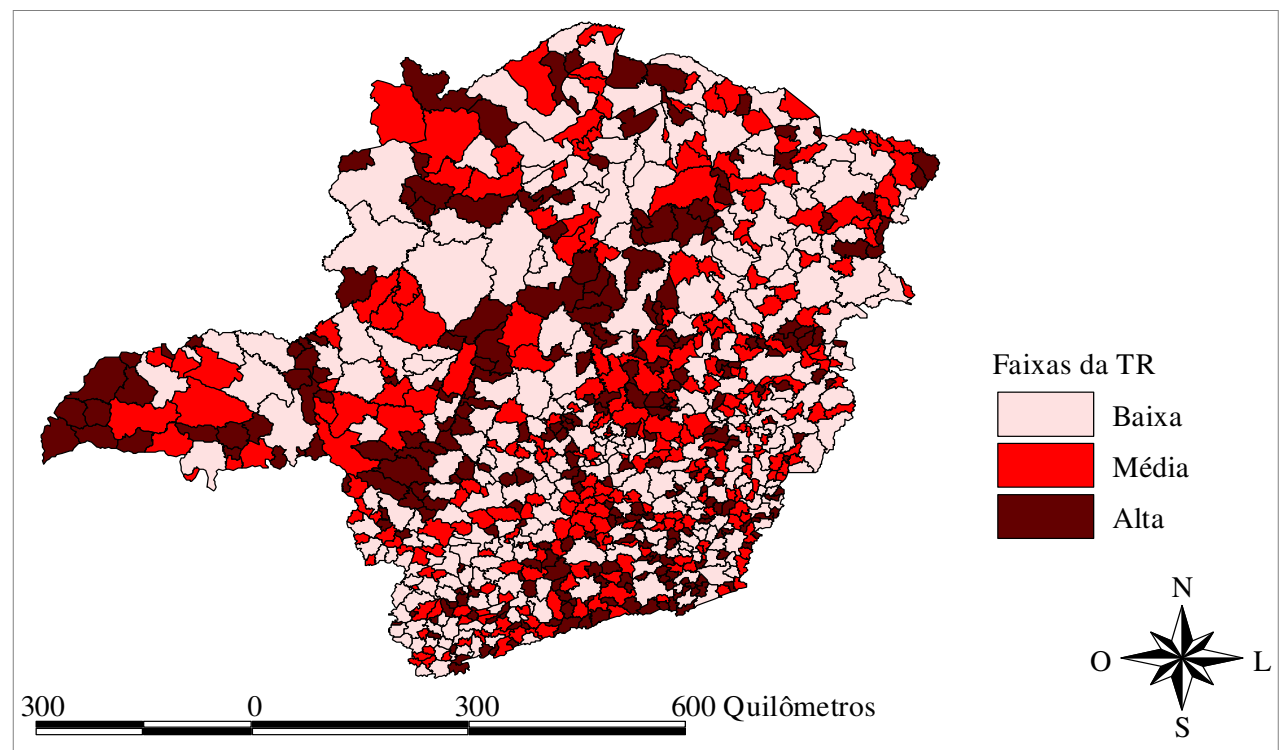

Observação: os valores plotados estão a preços reais do ano de 2004 e a variável sob análise está na forma per capita.

Fonte: Resultados da pesquisa.

O trabalho está dividido em cinco seções, incluída esta de caráter introdutório. A primeira seção trata dos dados; a segunda seção efetua uma análise exploratória dos dados espaciais; a terceira seção implementa a análise econométrica; a quinta seção reúne as considerações finais.

\section{Dados}

Os dados abrangem os 853 municípios de Minas Gerais, que são analisados no período entre 2001 e 2005 . É importante destacar que nesse período não houve a criação de novos municípios. Também nesse período, a repartição da cota-parte do ICMS para os municípios ficou sob os auspícios da mesma lei estadual (n. 3.803 de 27/12/2000), com os mesmos critérios e porcentuais praticados. Com efeito, embora se pudesse argumentar tratar-se de uma análise de curto prazo, para os presentes propósitos, a análise não fica prejudicada.

Os valores dos repasses da Lei Robin Hood, resultantes da aplicação dos critérios dispostos no Quadro 1, são o objeto de análise neste trabalho. A análise é do Repasse Líquido, ${ }^{5}$ pois se têm como descontados os valores destinados ao Fundef (Fundo de Manutenção e Desenvolvimento do Ensino Fundamental e de

(5) Essa informação foi obtida junto à Fundação João Pinheiro. 
Noé Gonçalves Maranduba Júnior / Eduardo Simões de Almeida

Valorização do Magistério) e ao Pasep (Programa de Formação do Patrimônio do Servidor Público).

Trabalha-se com essa variável per capita (doravante representada por TRPER), para que assim se tenha um padrão adequado de comparação dos valores entre os municípios. Quando se utilizam dados espaciais, como neste artigo, é recomendável que se adotem variáveis intensivas e não variáveis absolutas que podem ser influenciadas pelo tamanho da população ou da área geográfica. Ao se trabalhar com variáveis absolutas, aumenta a probabilidade de se ter correlações espaciais espúrias (Anselin, 2005).

Os dados sobre o Produto Interno Bruto (PIB) e a população municipal (estimativas das populações residentes) foram obtidos junto ao IBGE (Fundação Instituto Brasileiro de Geografia e Estatística).

\section{Análise exploratória de dados espaciais}

A utilização da análise exploratória de dados espaciais (Aede) se justifica por ser um método que objetiva descrever a distribuição espacial, os padrões de associação espacial (clusters espaciais), verificar a existência de diferentes regimes espaciais ou outras formas de instabilidade espacial (não estacionariedade) e identificar observações atípicas (i.e., outliers). Para implementá-la, assim como para aplicar as técnicas de econometria espacial, se faz necessário definir uma matriz de pesos espaciais $(W)$, que é a forma de expressar a estrutura espacial dos dados, sendo que os elementos $w_{i i}$ na diagonal são iguais a zero, enquanto os elementos $w_{i j}$ indicam a forma como a região $i$ está espacialmente conectada com a região $j$. A escolha da matriz de pesos espaciais é fundamental, pois os resultados da análise são sensíveis a essa seleção.

O critério de escolha da matriz de pesos espaciais baseou-se em verificar, conforme Baumont et al. (2002), para a variável sob análise, mediante a substituição de várias matrizes (rainha, torre, inverso da distância, inverso do quadrado da distância, $K$-vizinhos mais próximos, para $K=1, K=2, K=4, K=5$, $K=10, K=15, K=20$ e $K=25$ ), qual proporcionou o maior e mais significativo valor da estatística $I$ de Moran para os anos do período em análise. Mediante tal procedimento, a matriz do tipo $K=5$, normalizada na linha, foi selecionada para ser utilizada neste trabalho.

Um outro mecanismo muito útil para implementar a Aede e a econometria espacial é o operador de defasagem espacial. Mediante a utilização de pesos espaciais, pode-se obter uma medida da variável defasada para uma dada região, pois o operador de defasagem espacial de uma variável $y$, formalmente $W y$, pode ser interpretado como sendo a média do valor dessa variável nas regiões vizinhas. 
A autocorrelação espacial global pode ser calculada por meio da estatística I de Moran.

O I de Moran indica formalmente o grau de associação linear entre os vetores de valores observados no tempo $t\left(z_{t}\right)$ e a média ponderada dos valores da vizinhança, ou as defasagens espaciais $\left(W z_{t}\right)$. Valor de $I$ maior (ou menor) do que o valor esperado $E(I)=-1 /(n-1)$ significa que há autocorrelação positiva (ou negativa). Seguindo Cliff e Ord (1981), em termos formais, a estatística $I$ de Moran pode ser expressa como:

$$
I_{t}=\left(\frac{n}{S_{o}}\right)\left(\frac{z_{t}^{\prime} W z_{t}}{z_{t}^{\prime} z_{t}}\right) t=1, \ldots n
$$

em que $z_{t}$ é o vetor de $n$ observações para o ano $t$ na forma de desvio em relação à média. $W$ é a matriz de pesos espaciais. O termo $S_{o}$ é um escalar igual à soma de todos os elementos de $W$.

Quando a matriz de pesos espaciais é normalizada na linha, isto é, quando os elementos de cada linha somam 1, a expressão (1) fica da seguinte forma:

$$
I_{t}=\left(\frac{z_{t}^{\prime} W z_{t}}{z_{t}^{\prime} z_{t}}\right) t=1, \ldots n
$$

O I de Moran fornece três tipos de informação. A significância provê a informação se os dados estão distribuídos aleatoriamente ou não. O sinal positivo da estatística $I$ de Moran, desde que significativo, indica que os dados estão concentrados através dos municípios. O sinal negativo indica a dispersão dos dados. A magnitude da estatística fornece a força da associação espacial: o $I$ de Moran varia de -1 a +1 . Quanto mais próximo de um, mais forte é a concentração; quanto mais próximo de -1 , mais dispersos estão os dados.

A autocorrelação espacial, se for positiva, indica que municípios que apresentam elevados recebimentos de repasses da Lei Robin Hood são vizinhos de outros municípios que também apresentam elevados recebimentos desses repasses ou, alternativamente, que municípios com baixos recebimentos de repasses são circundados por outros municípios também ostentando baixos recebimentos desses repasses. A autocorrelação negativa tem uma interpretação inversamente análoga.

A Tabela 1 mostra os resultados dos testes de autocorrelação espacial para os repasses per capita da Lei Robin Hood (TRPER), respectivamente, para os anos de 2001 e de 2005. Como podem ser observados, os valores da estatística $I$ de Moran são positivos e crescentes, o que identifica uma autocorrelação espacial global positiva nos respectivos anos. 
Noé Gonçalves Maranduba Júnior / Eduardo Simões de Almeida

Tabela 1

Teste de autocorrelação espacial para TRPER

\begin{tabular}{c|c|c|c|c|c}
\hline Ano & $I$ de Moran & Média & Desvio Padrão & Valor de Z & Prob. \\
\hline 2001 & 0.119 & -0.001 & 0.021 & 5.793 & 0.000 \\
\hline 2005 & 0.145 & -0.001 & 0.021 & 7.039 & 0.000 \\
\hline
\end{tabular}

Fonte: Resultados da pesquisa.

A estatística $I$ de Moran revela que essas variáveis seguem um padrão espacial não aleatório. Municípios que recebem altos repasses tendem a estar rodeados por municípios que recebem altos repasses, e municípios com baixos repasses tendem a estar rodeados por municípios com baixos repasses.

Para refinar os resultados do teste de autocorrelação espacial global, é preciso calcular a chamada estatística Lisa (Anselin, 1995). Segundo Le Gallo e Ertur, apud Perobelli et al. (2006), a estatística Lisa, baseada no I de Moran local pode ser especificada da seguinte forma:

$$
I_{i, t}=\frac{\left(x_{i, t}-\mu_{t}\right)}{m_{o}} \sum_{j} w_{i j}\left(x_{j, t}-\mu_{t}\right) \operatorname{com} m_{o}=\frac{\left(x_{i, t}-\mu_{t}\right)^{2}}{n}
$$

na qual $x_{i, t}$ é a observação de uma variável de interesse na região $i$ para o ano $t$, $\mu_{t}$ é a média das observações entre as regiões no ano $t$ para a qual o somatório em relação a $j$ é tal que somente os valores vizinhos de $j$ são incluídos.

A estatística pode ser interpretada da seguinte maneira: valores positivos de $I_{i, t}$ indicam que há clusters espaciais com valores similares (alto ou baixo); valores negativos significam que há clusters espaciais com valores diferentes entre as regiões e seus vizinhos. Considerando que é grande o número das informações computadas, o ideal é mapeá-las, formando o chamado mapa de clusters. Feito isso, têm-se os mapas expressos nas Figuras 2 e 3, respectivamente, para os anos de 2001 e 2005.

Por meio dos mapas de clusters (Figuras 2 e 3), que georreferenciam os resultados da estatística $I_{i}$ significativos no nível de $95 \%$ de confiança, podem-se apurar os regimes espaciais mais interessantes para TRPER01 e TRPER05. Notase a presença do padrão alto-alto (AA) no Triângulo Mineiro e Alto Paranaíba, na parte Central e Mata mineiras. Os padrões baixo-baixo (BB) se encontram no Norte de Minas, Jequitinhonha e Mucuri. O padrão baixo-alto (BA) pode ser apreciado em diversas regiões do estado, destacando-se no Triângulo Mineiro, Alto Paranaíba e Mata. Enfim, notam-se algumas pequenas alterações nos padrões de organização espacial, ao longo do período sob análise. 
Figura 2

Mapa de Clusters para TRPER01

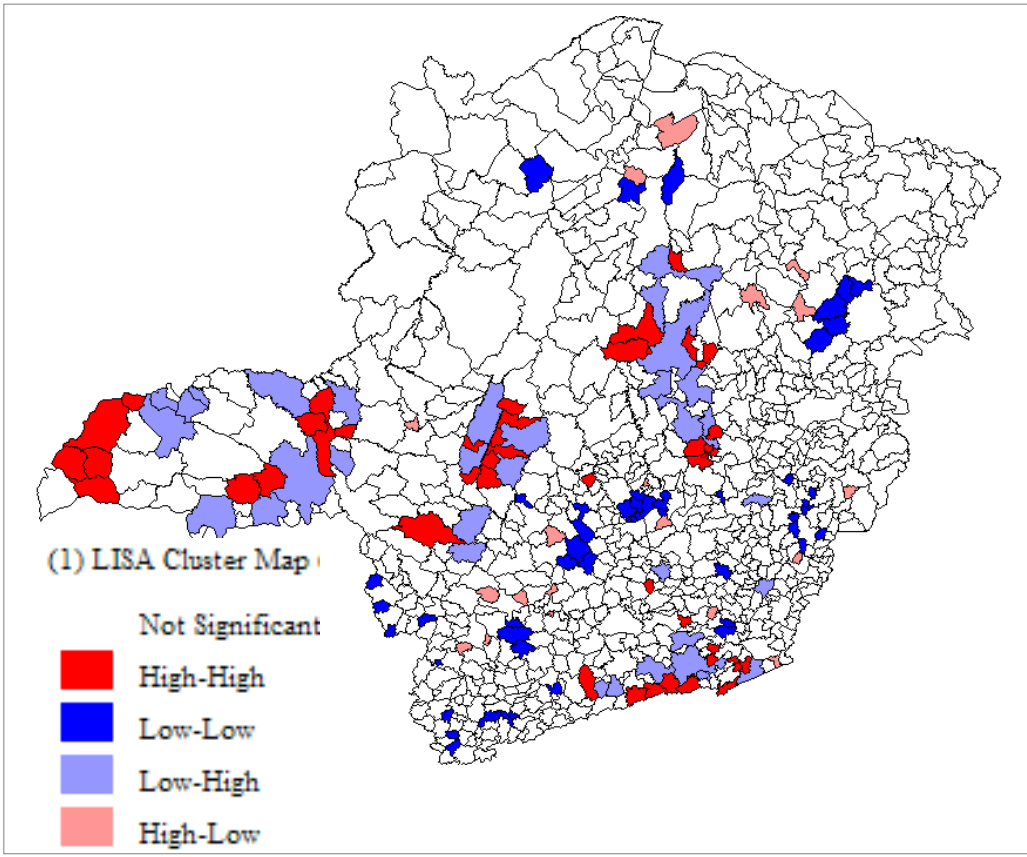

Fonte: Resultados da pesquisa.

Figura 3

Mapa de Clusters para TRPER05

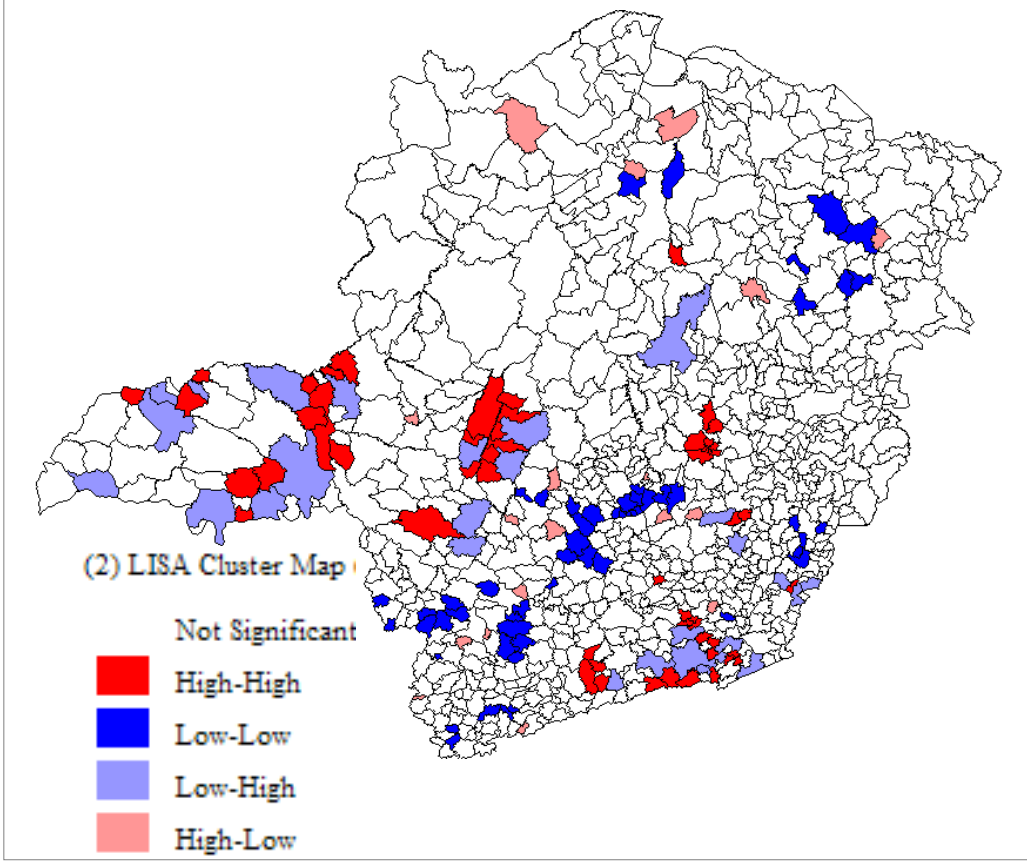

Fonte: Resultados da pesquisa. 
Noé Gonçalves Maranduba Júnior / Eduardo Simões de Almeida

Martins (1998), em suas análises de Minas Gerais, demonstrou, por intermédio de alguns indicadores regionais, que as regiões Central, Mata, Sul e Triângulo são as mais desenvolvidas em termos econômicos e sociais. Já as regiões Norte, Jequitinhonha/Mucuri e Noroeste são aquelas problemáticas. Assim, os padrões espaciais aqui encontrados demonstram sintonia com os padrões socioeconômicos do Estado.

Em síntese, os resultados da Aede revelam que os valores repassados aos 853 municípios mineiros, conforme a Lei Robin Hood, não foram distribuídos aleatoriamente, devido à existência da autocorrelação espacial positiva nos dados. A heterogeneidade espacial, representada tanto pela associação espacial local positiva (clusters espaciais com municípios AA e BB) como pela associação espacial local negativa (clusters espaciais com municípios $\mathrm{AB}$ e $\mathrm{BA}$ ) é identificada. A análise foi feita com base em duas fotografias tiradas em dois pontos distintos do tempo. Tal análise é essencialmente estática.

Para detectar se é verdadeira a hipótese de a Lei Robin Hood ter apresentado efeitos redistributivos ao longo do período, aprofundando a investigação, é preciso realizar uma análise de convergência, que permite apurar dinamicamente se os repasses para os municípios mais pobres cresceram a taxas superiores às dos repasses para os municípios mais ricos.

\section{Análise de convergência espacial}

A análise de convergência é um instrumental que vem encontrando larga escala de aplicações. No caso brasileiro, a literatura abordando o tema da convergência de renda entre estados e regiões é vasta, conforme destaca Maranduba Jr. (2007). Segundo esse autor, podem-se dividir os estudos em dois tipos: 1) aqueles que não utilizaram os recursos analíticos da econometria espacial (Fontes; Alves, 2000; Silva et al., 2004; Pinto, 2005; Wanderley, 1997 apud Pinto, 2005; Nunes; Nunes, 2005; Souza; Porto, 2006; Azzoni; Neto, 2006; Azzoni et al., 2006; Oliveira et al., 2006; Pinto Coelho, 2006; Salvato et al., 2006 e Fochezatto; Stulp, 2006); 2) aqueles que utilizaram os recursos da econometria espacial (Magalhães et al., 2000; Magalhães, 2001; Monastério; Ávila, 2004; Pimentel; Haddad, 2004; Resende, 2005; Oliveira, 2005; Silva; Resende, 2006; Perobelli et al., 2006; Barreto, 2007 e Maranduba Jr., 2007).

De acordo com Barro e Sala-i-Martin (1999), a chamada convergência absoluta considera que as economias atrasadas tendem a crescer a taxas mais elevadas do que as economias ricas e que, portanto, em algum momento do tempo, as economias pobres acabariam alcançando o nível de renda per capita das 
economias ricas. Essa noção de convergência requer que se suponha que tanto as economias ricas como as pobres possuem idênticas tecnologias (ou têm livre acesso às mesmas), idênticas preferências, idênticas instituições políticas e outras características econômicas.

A maneira tradicional de se calcular a convergência absoluta (ou $\beta$ convergência) consiste em se regredir a taxa de crescimento da renda ou produto per capita em relação ao logaritmo da renda ou produto per capita regional inicial. A convenção tem sido interpretar uma estimativa negativa para $\beta$ como apoio à hipótese de convergência, desde que tal estimativa sugeriria que as taxas de crescimento de renda per capita ao longo de alguns anos fossem negativamente correlacionadas com as rendas iniciais. Em outros termos, seria como dizer que uma variável com valores distintos entre regiões teria suas diferenças diminuídas com o decorrer do tempo.

Conforme Oliveira et al. (2006), a aplicação para municípios de uma metodologia desenvolvida para análise de convergência de países merece algumas ressalvas tanto do ponto de vista teórico quanto empírico. Se, por um lado, municípios de um mesmo estado apresentam características semelhantes, pois possuem a mesma política econômica, compartilham de algumas instituições, possuem atividades econômicas afins etc., por outro lado, fatores como mobilidade de capitais e de mão de obra permitem a aglomeração das atividades em alguns municípios do estado em detrimento de outros, não sendo incomum, portanto, a existência de grandes desigualdades dentro de um mesmo estado.

No presente trabalho, testa-se se houve convergência dos repasses da Lei Robin Hood aos municípios mineiros, no período de 2001 a 2005. Busca-se auferir, assim, se os municípios mais pobres passaram a receber mais daqueles recursos. Tendo-se em conta os critérios da Lei Robin Hood (vistos no Quadro 1), interpreta-se que taxas de crescimento dos repasses maiores para municípios que recebiam menos no início do período indicam a realização de esforços socioeconômicos pelos respectivos municípios, o que poderia ser um indicativo favorável à redução das desigualdades regionais.

A implementação de um modelo que procure captar questões inerentes a "efeitos de transbordamentos" entre unidades espaciais deve considerar explicitamente componentes espaciais em sua forma funcional. Assim, os modelos tradicionais de convergência e a aplicação aos repasses dos tributos não podem ser estimados por intermédio do método dos mínimos quadrados ordinários (MQO), pois as estimativas serão inconsistentes e/ou ineficientes. 
Noé Gonçalves Maranduba Júnior / Eduardo Simões de Almeida

Assim sendo, ao se estimar por MQO o modelo de $\beta$ convergência, buscase apenas identificar qual é a melhor maneira de estimar a equação que é dada por:

$$
\operatorname{Ln}[\text { TRPER05/TRPER01 }]_{\mathrm{i}}=\alpha_{\mathrm{i}}+\beta \ln [\text { TRPER01 }]_{\mathrm{i}}+\mu_{\mathrm{i}}
$$

em que Ln [TRPER05/TRPER01] i é o logaritmo natural da razão entre os valores de repasses efetuados aos municípios (neste caso, as variáveis estão per capita) de acordo com critérios da Lei Robin Hood, entre os anos 2001 e 2005. Por sua vez, $\ln$ [TRPER01] é o logaritmo natural dos repasses efetuados no ano-base (2001). Finalmente, $\mu_{\mathrm{i}}$ é o termo de erro aleatório e $i$ é o indexador para os diversos municípios.

A fim de identificar a melhor especificação do modelo de $\beta$ convergência segue-se a proposta feita por Florax; Folmer e Rey (2003), isto é, numa primeira etapa, seguir o seguinte roteiro:

a) Estimar o modelo clássico de análise de regressão linear por MQO;

b) Testar a hipótese de ausência de autocorrelação espacial devido a uma defasagem ou a um erro por meio das estatísticas Multiplicador de Lagrange (ML) $\rho$ (defasagem espacial) e Multiplicador de Lagrange (ML) $\lambda$ (erro espacial);

c) Caso ambos os testes não sejam significativos, a utilização do modelo clássico é mais apropriada. Caso contrário, é necessário seguir o próximo passo;

d) Caso ambos sejam significativos, estima-se o modelo apontado como o mais significante de acordo com as versões robustas desses testes, ou seja, Multiplicador de Lagrange Robusto (MLR) $\rho$ (defasagem espacial) e Multiplicador de Lagrange Robusto (MLR) $\lambda$ (erro espacial). Assim, caso MLR $\rho$ $>$ MLR $\lambda$, usa-se o modelo com defasagem espacial como o mais apropriado. Caso contrário, MLR $\rho<\operatorname{MLR} \lambda$, adota-se o modelo de erro autorregressivo como o mais apropriado.

Assim sendo, se for rejeitada a hipótese de ausência de autocorrelação espacial, devem-se adotar os procedimentos apropriados, ou seja, especificar a equação de $\beta$ convergência por meio dos modelos mais adotados nas aplicações de econometria espacial, quais sejam: o modelo de erro espacial, o modelo de defasagem espacial ou o modelo regressivo cruzado espacial.

A estimação da equação (4) permite obter a estimativa do parâmetro da convergência $\beta$, que estuda a hipótese de que os recebimentos de municípios com repasses baixos tendem a crescer mais rapidamente que os recebimentos de municípios com repasses maiores, alcançando-os.

Ao seguir o roteiro dado anteriormente, estima-se a equação (4) por MQO. Os resultados estão reportados na Tabela 2, adiante. Ressalte-se que os testes para averiguar a presença de autocorrelação espacial são úteis tanto para servir de 
auxílio no momento de identificação do modelo econométrico espacial quanto para a tarefa de validação ou diagnóstico desse modelo.

Cabe informar a possibilidade de dois problemas ocorrerem nos modelos estimados: a existência de heterocedasticidade, conforme pode ser verificado pelos testes Breusch-Pagan, Koenker-Bassett e White; e os erros não serem normais, de acordo com o teste Jarque-Bera; caso ocorram, ambos os problemas colocam em suspeita as inferências.

Na Tabela 2, na coluna "sem dummies", observa-se que o modelo estimado apresentou o problema da não normalidade dos erros, conforme teste de Jarque-Bera e, ainda, apresentou o problema de heterocedasticidade, conforme se confirma pelo teste de Koenker-Bassett e pelo teste de White.

Objetivando corrigir a ausência de normalidade dos erros, adotou-se o recurso de, a partir dos resíduos da regressão estimada por MQO, efetuar o mapeamento dos mesmos, sendo que foram colocadas duas variáveis dummies, referentes aos termos de erros que se evidenciaram outliers superiores (DOTSUP) e outliers inferiores (DOTINF), detectados com base no Box Plot, com a definição de um hinge no valor de 1,5 .

Assim, na Tabela 2, na coluna "com dummies", encontram-se as estimativas dos coeficientes e probabilidades respectivas. O teste de Jarque-Bera agora permite a hipótese de que os resíduos sejam distribuídos aleatoriamente; no entanto, o teste de Breusch-Pagan rejeita a hipótese de homocedasticidade. Não houve problemas de multicolinearidade. Com efeito, há que se resolver ainda o problema da heterocedasticidade. Ao considerarem-se os critérios de informação Akaike Information Criterion (AIC), Schwarz Criterion (SC) e do valor da função de verossimilhança (LIK), conforme podem ser observados na Tabela 2, o modelo com dummies se revela mais apropriado.

A hipótese de ausência de autocorrelação espacial devido a uma defasagem ou a um erro por meio das estatísticas Multiplicador de Lagrange (ML) lag e Multiplicador de Lagrange (ML) error é rejeitada pelos respectivos testes, num nível de significância que aponta para a necessidade das versões robustas desses testes.

As versões robustas desses testes apontam para a existência da autocorrelação espacial devido a uma defasagem, num nível de significância de $10 \%$. Assim, o modelo indicado pelos testes é o modelo de defasagem espacial.

Todos os coeficientes são significativos, à exceção do coeficiente $\beta$, assim como têm os sinais esperados. 
Noé Gonçalves Maranduba Júnior / Eduardo Simões de Almeida

Tabela 2

Resultados econométricos para o Modelo de Convergência

\begin{tabular}{|c|c|c|}
\hline & $\begin{array}{c}2001 / 2005 \\
\text { (sem dummies) }\end{array}$ & $\begin{array}{c}2001 / 2005 \\
\text { (com dummies) }\end{array}$ \\
\hline \multirow{2}{*}{$\alpha$} & 0.612806 & 0.5429 \\
\hline & $(0.000000)$ & $(0.000000)$ \\
\hline \multirow{2}{*}{$\beta$} & -0.0216173 & -0.0035837 \\
\hline & $(0.073832)$ & $(0.731958)$ \\
\hline \multirow{2}{*}{ Dotsup } & \multirow[b]{2}{*}{-} & 0.554016 \\
\hline & & $(0.000000)$ \\
\hline \multirow{2}{*}{ Dotinf } & \multirow[b]{2}{*}{-} & -0.629183 \\
\hline & & $(0.000000)$ \\
\hline \multirow{2}{*}{ I de Moran } & 0.088912 & 0.072201 \\
\hline & $(0.000000)$ & $(0.00000)$ \\
\hline \multirow{2}{*}{ ML - Erro } & 25.268765 & 16.6629 \\
\hline & $(0.000000)$ & $(0.000045)$ \\
\hline \multirow{2}{*}{ ML - defasagem } & 21.605151 & 19.045013 \\
\hline & $(0.000003)$ & $(0.000013)$ \\
\hline \multirow{2}{*}{ MLR - Erro } & 36.729731 & 0.3422 \\
\hline & $(0.000000)$ & $(0.558539)$ \\
\hline \multirow{2}{*}{ MLR - Defasagem } & 33.066116 & 2.7244 \\
\hline & $(0.000000)$ & $(0.098826)$ \\
\hline \multirow{2}{*}{ Teste de White } & 12.811726 & \multirow[b]{2}{*}{-} \\
\hline & $(0.001652)$ & \\
\hline \multirow{2}{*}{ Teste de Koenker-Bassett } & 12.694864 & \multirow{2}{*}{-} \\
\hline & $(0.000367)$ & \\
\hline \multirow{2}{*}{ Teste de Breusch-Pagan } & \multirow[t]{2}{*}{ (5) } & 13.1049 \\
\hline & & $(0.004415)$ \\
\hline \multirow{2}{*}{ Teste de Jarque - Bera } & 120.078759 & 6.174886 \\
\hline & $(0.000000)$ & $(0,045618)$ \\
\hline AIC & -340.949 & -600.8400 \\
\hline $\mathrm{SC}$ & -331.452 & -581.8450 \\
\hline LIK & 172.475 & 304.4200 \\
\hline
\end{tabular}

Nota: os resultados entre parênteses indicam a probabilidade. A matriz de pesos espaciais utilizada foi a de $\mathrm{k}=5$ vizinhos mais próximos.

Fonte: Resultados da pesquisa.

Após seguir os critérios já explicitados, foi possível estimar o modelo de convergência absoluta, ${ }^{6}$ tal como pode ser observado na Tabela 3, a seguir. Considerando-se a não normalidade dos resíduos e a heterocedasticidade, estimouse pelo método dos mínimos quadrados em dois estágios (MQ2E) e pelo método

(6) Antes, porém, vale registrar que sob a suposição de normalidade dos resíduos, estimou-se tanto o modelo de defasagem quanto o modelo de erro espacial por máxima verossimilhança, sendo que o critério do valor da função de verossimilhança (LIK) indicou a melhor qualidade de ajustamento para o primeiro modelo, confirmando o indicativo do teste Multiplicador de Lagrange. Ambos os modelos apresentaram problemas de heterocedasticidade. Frise-se que em todos eles (incluído aí o modelo de erro espacial estimado por GMM, conforme procedimento proposto por Kelejian e Prucha) o coeficiente de $\beta$ apresentou sinal negativo, mas não significativo a 55\%. Sob o pressuposto de não normalidade, estimou-se por mínimos quadrados em dois estágios (inclusive na versão robusta). 
dos mínimos quadrados em dois estágios na versão robusta (haja vista que neste utiliza-se a matriz de variância-covariância de White na estimação, permitindo-se que os desvios-padrão sejam estimados consistentemente).

Tabela 3

Resultados econométricos do modelo de convergência espacial

\begin{tabular}{|c|c|c|}
\hline & $\begin{array}{l}\text { Modelo de Defasagem } \\
\text { Espacial (MQ2E) }\end{array}$ & $\begin{array}{c}\text { Modelo de Defasagem } \\
\text { Espacial (MQ2E Robusto) }\end{array}$ \\
\hline \multirow[b]{2}{*}{$\alpha$} & 0.388454 & 0.391291 \\
\hline & $(0.00000)$ & $(0.00000)$ \\
\hline \multirow[b]{2}{*}{$\beta$} & -0.005257 & -0.005699 \\
\hline & $(0.610682)$ & $(0.566381)$ \\
\hline \multirow{2}{*}{ Dotsup } & 0.54895 & 0.549157 \\
\hline & $(0.00000)$ & $(0.00000)$ \\
\hline \multirow{2}{*}{ Dotinf } & -0.610712 & -0.609907 \\
\hline & $(0.00000)$ & $(0.00000)$ \\
\hline \multirow[b]{2}{*}{$\rho$} & 0.301890 & 0.299945 \\
\hline & $(0.001406)$ & $(0.000522)$ \\
\hline \multirow{2}{*}{ Lagrange Multiplier (error) } & 2.564091 & \multirow{2}{*}{-} \\
\hline & $(0.109315)$ & \\
\hline
\end{tabular}

Nota: Os resultados entre parênteses indicam a probabilidade. A matriz de pesos espaciais utilizada foi a de $\mathrm{k}=5$ vizinhos mais próximos.

Fonte: Resultados da pesquisa.

As estimativas constantes na Tabela 3, relativas ao modelo de defasagem espacial, se revelam altamente significativas. O teste do Multiplicador de Lagrange para a dependência no termo de erro indica que esta não é importante, no nível de $10 \%$. Com efeito, o modelo de defasagem espacial apresentou um bom ajustamento. Na versão robusta, o programa não fornece estatísticas para se avaliar a dependência espacial remanescente. Nessa versão, observa-se que todos os coeficientes permanecem com o mesmo sinal e apenas levemente alterados em seus valores, não comprometendo a qualidade da análise.

Embora o sinal da estimativa do parâmetro $\beta$ nos modelos seja negativo, torna-se possível observar a insignificância estatística desse coeficiente, sugerindo que ele não exerce nenhuma influência sobre a variável dependente. De forma prática, isso informa que os repasses da Lei Robin Hood, com os atuais critérios de distribuição, não estão contribuindo para que municípios mais pobres do Estado recebam mais recursos provenientes de municípios mais ricos. Isso indica a necessidade de que o governo do estado de Minas Gerais reveja os critérios redistributivos da Lei Robin Hood, de forma que acelere as taxas de repasses para os municípios mais pobres. Do contrário, o que se pode supor é que, dados aqueles critérios, a tendência é a da concentração dos recursos nos municípios mais ricos, cabendo a indagação de se seria apropriada a denominação de Lei Robin Hood (que retira dos que têm mais para dar aos que têm menos). 
Noé Gonçalves Maranduba Júnior / Eduardo Simões de Almeida

\section{Considerações finais}

Neste trabalho, foi possível prover evidências sobre o comportamento temporal e espacial dos repasses da Lei Robin Hood. Há indicativos em favor da inexistência da convergência nos repasses efetuados entre 2001 e 2005. Isso sinaliza que, com o objetivo de amenizar questões relativas à heterogeneidade socioeconômica dos municípios mineiros, torna-se necessário repensar os critérios redistributivos desses recursos.

Talvez critérios mais simples promovam uma melhor distribuição dos recursos fiscais da Lei Robin Hood dentro do seu espírito original, ou seja, repassar recursos de municípios ricos para os municípios mais pobres. Um critério simples para atingir esse fim seria o inverso da renda per capita: municípios com um nível de renda per capita abaixo da média do estado de Minas Gerais receberiam mais que proporcionalmente do que os municípios mais ricos. Tal critério está em consonância com o espírito dos fundos regionais criados dentro da União Europeia para promover a equidade da renda per capita entre as regiões da Europa. Se se quer diminuir as discrepâncias da renda regional, nada melhor do que usar como critério de distribuição a própria renda.

Os resultados deste artigo provêm valiosos subsídios para o debate que se trava atualmente na Assembleia Legislativa de Minas Gerais a respeito da revisão dos critérios de repasse dos recursos da Lei Robin Hood.

Uma das possibilidades futuras deste trabalho, tendo em vista que a preocupação aqui foi analisar a distribuição espacial e a convergência dos recursos da Lei Robin Hood, é abrir a análise para cada um dos critérios, conforme dispostos no Quadro 1. Com efeito, seriam analisados os repasses pelo critério de educação, de saúde, dentre outros. Se o intuito é redistribuir rendas, mediante transferências constitucionais, poder-se-ia propor, no atual contexto de uma reforma tributária, uma revisão da adequação dos repasses de forma a serem desvinculados do critério VAF.

\section{Referências bibliográficas}

ALMEIDA, E. S. de. Econometria espacial aplicada. Mestrado (Economia Aplicada)Universidade Federal de Juiz de Fora, 2006. Mimeografado.

ANSELIN, Luc. Spatial econometrics: methods and models. Boston: Kluwer Academic, 1988.

Local indicators of spatial association - LISA. Geographical Analysis. v. 27, n. 2, p. 93-115, Apr. 1995.

Exploring spatial data with GeoDa: a workbook. Urbana-Champaign: University of Illinois, 2005. 
AZZONI, C. R.; NETO, R. M. S. Radiografando a convergência regional: fontes setoriais e mudanças estruturais. Disponível em: http://www.nemesis.org.br/docs/rau11.pdf. Acesso em: 31 out. 2006.

; MENEZES, Tatiane A.; SANTOS, A. M. S. P. Convergência de renda real e nominal entre as regiões metropolitanas brasileiras: uma análise de dados de painel. Disponível em: http://www.nemesis.org.br/docs/tatiane1.pdf. Acesso em: 15 nov. 2006.

BARRETO, R. C. S. Desenvolvimento regional e convergência de renda nos municípios do Estado do Ceará. Tese (Doutorado)-Universidade Federal de Viçosa, Minas Gerais, 2007.

BARRO, Robert J.; SALA-I-MARTIN, Xavier. Economic growth. Cambridge, Massachusetts: The MIT Press/London, England: First MIT Press Edition, 1999.

BAUMONT, C.; ERTUR, C.; LE GALLO, J. The European regional convergence process, 1980-1995: do spatial regimes and spatial dependence matter? University of Burgundy, Jul. 2002. Disponível em: http://129.3.20.41/eos/em/papers/0207/0207002.pdf. Acesso em: 10 mar. 2007.

CLIFF, A.; ORD, J. Spatial processes, models and applications. London: Pion, 1981.

FLORAX, Raymond J. G. M.; FOLMER, Hendrik; REY, Sergio J. Specification searches in spatial econometrics: The relevance of Hendry's methodology. Regional Science and Urban Economics, v. 33, n. 5, p. 557-579, 2003.

FOCHEZATTO, A.; STULP, V. J. Análise da convergência da renda per capita entre os municípios do Rio Grande do Sul, 1985-1998. Disponível em: http://www.pucrs.br/notfound.htm?pagina=/eventos/3eeg/Artigos/m23t03.pdf. Acesso em: 15 dez. 2006.

FONTES, R.; ALVES, L. F. Clubes de convergência entre os municípios de Minas Gerais. In: SEMINÁRIO SOBRE A ECONOMIA MINEIRA, 9, 2000. Disponível em http://www.cedeplar.ufmg.br/diamantina2000/2000/FONTES.pdf. Acesso em: $10 \mathrm{fev}$. 2007.

FUNDAÇÃO JOÃO PINHEIRO. Índice Mineiro de Responsabilidade Social. dez. 2005. (Versão 1.0. CD-ROM).

IBGE. INSTITUTO BRASILEIRO DE GEOGRAFIA E ESTATÍSTICA. Disponível em: http://www.ibge.gov.br/home/estatistica/economia/pibmunicipios/2005/default.shtm.

Acesso em: 05 jun. 2008.

MAGAlhãeS, A.; HEWINGS, G. J. D.; AZZONI, C. R. Spatial dependence and regional convergence in Brazil. Urbana Champaign, 2000. (Working Paper REAL, 00-T$11)$.

MAGALHÃES, A. M. Clubes de convergência no Brasil: uma abordagem com correção espacial. In: ENCONTRO NACIONAL DE ECONOMIA, 29, 2001, Salvador. Anais... Disponível em: http://www.anpec.org.br/encontro2001/artigos/200105056.pdf. Acesso em: 15 dez. 2006. 
Noé Gonçalves Maranduba Júnior / Eduardo Simões de Almeida

MARANDUBA JR., N. G. Política regional, crescimento econômico e convergência de renda em Minas Gerais. Dissertação (Mestrado)- Faculdade de Economia e Administração da Universidade Federal de Juiz de Fora, Juiz de Fora, MG, 2007.

MARTINS, Nubia M. de A. A Lei Robin Hood como instrumento para a redução das heterogeneidades das regiões mineiras: análise de seus objetivos e resultados através de um exercício de convergência. Monografia (Curso de Economia)-Universidade Federal de Juiz de Fora, set. 1998.

MINAS GERAIS. AL debate ICMS solidário no interior. Diário Oficial do Estado de Minas Gerais. Imprensa Oficial, Belo Horizonte, 7 ago. 2007. p. 2.

MONASTÉRIO, L.; ÁVILA, R. Uma análise espacial do crescimento econômico do Rio Grande do Sul (1939-2001). In: ENCONTRO NACIONAL DE ECONOMIA, 2004. Anais... Anpec.

NUNES, Ricardo da C.; NUNES, Selene P. P. O papel dos Fundos de Participação dos Estados - FPE na convergência da renda per capita dos estados brasileiros. Disponível em: http://eco.uncor.edu/ief/publicaciones/economestadistica/2004_42_n2/5_nunes.pdf. Acesso em: 15 nov. 2006.

OLIVEIRA, C. A. Externalidades espaciais e o crescimento econômico das cidades do estado do Ceará. Revista Econômica do Nordeste, Fortaleza, v. 36, n. 3, 21p, 2005.

; JACINTO, P. A.; GROLLI, P. A. Crescimento econômico e convergência com a utilização de regressões quantílicas: um estudo para os municípios do Rio Grande do Sul (1970-2001). Disponível em: http://www.pucrs.br/eventos/3eeg/Artigos/m23t02.pdf. Acesso em: 13 nov. 2006.

PEROBELli, F. S.; FARIA, W. R.; FERREIRA, P. G. C. Análise de convergência espacial no Estado de Minas Gerais: 1975-2003. In: ENCONTRO REGIONAL DE ECONOMIA/NORDESTE: ESTRATÉGIAS DE DESENVOLVIMENTO REGIONAL, 11, 2006, Fortaleza. Anais... Fortaleza: BNB/ANPEC, 2006.

PIMENTEL, E. A.; HADDAD, E. A. Análise da distribuição espacial da renda no Estado de Minas Gerais: uma abordagem setorial. 2004. Disponível em: http://www.econ.fea.usp.br/nereus/td/Nereus_02_04.pdf. Acesso em: 11 out. 2006.

PINTO, E. C. L. Desigualdades econômicas regionais no Estado da Bahia entre 1970 e 1998: um teste da hipótese de Convergência de Renda. Dissertação (Mestrado)Universidade Federal da Bahia, Salvador, 2005.

PINTO COELHO, R. L. Dois ensaios sobre a desigualdade de renda dos municípios brasileiros. Dissertação (Mestrado)- Centro de Desenvolvimento e Planejamento Regional da Faculdade de Ciências Econômicas, UFMG, Belo Horizonte, nov. 2006.

RESENDE, G. M. Testes de robustez e externalidades espaciais: o caso dos estados brasileiros e dos municípios mineiros. Dissertação (Mestrado)- Centro de Desenvolvimento e Planejamento Regional da Faculdade de Ciências Econômicas, UFMG, Belo Horizonte, 2005. 
REY, Sergio J.; MONTOURI, Brett D. US regional income convergence: a spatial econometrics perspective. Regional Studies, v. 33, n. 2, p. 143-156, 1999.

RIBEIRO, Eduardo P.; PORTO JR., Sabino da S. Distribuição espacial da renda per capita e crescimento entre os Municípios da região Nordeste do Brasil - uma análise markoviana. Disponível em: http://www.ufrgs.br/decon/virtuais/sabino/textos.htm. Acesso em: 1 nov. 2006.

SALVATO, M. A.; RAAD, R. J.; ARAÚJO JUNIOR, A. F.; MORAIS, F. Disparidades regionais em Minas Gerais. 2006. (Ibmec MG Working Paper, WP35).

SILVA, A. M. A.; RESENDE, G. M. Crescimento econômico comparado dos municípios alagoanos e mineiros: uma análise espacial. Brasília, fev. 2006. (Texto para discussão, n. 1162).

SILVA, E.; FONTES, R.; ALVES, L. F. Análise das disparidades regionais em Minas Gerais. In: SEMINÁRIO SOBRE A ECONOMIA MINEIRA,11, 2004, Diamantina. Anais...

SOUZA, Nali de J. de; PORTO JR., Sabino da S. Crescimento regional e novos testes de convergência para os municípios da região Nordeste do Brasil. Disponível em http://www.ufrgs.br/decon/virtuais/sabino/textos.htm. Acesso em: 1 nov. 2006. 